\title{
Cuestiones Ambientales y Seguridad Alimentaria en el Cantón Guano, Ecuador
}

\author{
Claudia M. Andrade ${ }^{(1)}$ y Víctor D. Ayaviri(2) \\ (1) Facultad de Recursos Naturales, Instituto de Posgrado y Educación Continua, Escuela Superior \\ Politécnica de Chimborazo, Riobamba-Ecuador (e-mail:claudimari8873@yahoo.es) \\ (2) Facultad de Ciencias Políticas y Administrativas, Universidad Nacional de Chimborazo, Riobamba- \\ Ecuador (vdayaviri@gmail.com)
}

Recibido Mar. 15, 2017; Aceptado May. 17, 2017; Versión final Jul. 27, 2017, Publicado Oct. 2017

\begin{abstract}
Resumen
La presente investigación tiene como objetivo estudiar las cuestiones ambientales que afectan la producción del cultivo de papa (solanum tuberosum) y su relación con la seguridad alimentaria del cantón Guano, Ecuador. Para ello, se aplicó una encuesta dirigida a la población rural para construir un modelo de regresión lineal simple, que se complementa con observación de campo y entrevistas. Los principales hallazgos son destrucción de páramos, incremento de plagas y enfermedades en los cultivos, contaminación ambiental con basura doméstica y heladas. Las causas que originaron los cambios ambientales mencionados son el uso excesivo de pesticidas, falta de tratamiento de basura doméstica, monocultivo y la deforestación provocada por el crecimiento de la frontera agrícola. Se concluye que los cambios ambientales han afectado la producción de papa, pero no determina ni compromete la seguridad alimentaria de la población.
\end{abstract}

Palabras clave: cuestiones ambientales; producción de papa; seguridad alimentaria; alimentos

\section{Environmental Issues and Food Security in Guano, Ecuador}

\begin{abstract}
The objective of this research was to study how the environmental issues affect the production of potato (solanum tuberosum) and its relationship with food security in Guano-Ecuador. To do this, a survey was conducted on a rural population in order to construct a simple linear regression model, which was complemented by field observations and interviews. The main findings were moorland's destruction, increase of pests and diseases in the crops, environmental contamination with domestic waste, and frost. These environmental changes were the result of the excessive use of pesticides, lack of domestic waste treatment, monoculture, and deforestation caused by the growth of the agricultural frontier. In summary, the environmental changes have affected potato production, but they do not determine or compromise the food security of the population.
\end{abstract}

Keywords: environmental issues; potatoes production; food security; population; foods 


\section{INTRODUCCION}

La Comisión Mundial sobre Ambiente y Desarrollo en 1983 llegó a la conclusión de que para satisfacer las necesidades del presente sin comprometer la capacidad de las futuras generaciones, la protección del medio ambiente y el crecimiento económico, habrían de abordarse como una sola cuestión, al respecto Correa (2004), indica que durante las últimas décadas, el crecimiento económico se ha dado, gracias a un incremento en el uso de energía y mayor uso de los recursos naturales, lo que ha provocado que las empresas presenten un interés en temas relacionados con la protección del medio ambiente (De Burgos y Céspedes, 2001). Así, al relacionar las dimensiones estructurales y coyunturales del capitalismo actual, así como, sus manifestaciones en el campo ambiental, partiendo de que las desigualdades antagónicas entre las clases sociales, las confronta en la pugna por el acceso y manejo de los recursos naturales y se afirma que el modelo de desarrollo extractivo y depredador, considera a la naturaleza como otra mercancía para capitalizar (Franceschi, 2014; Machin y Casas, 2006). Por lo que, el análisis de los cambios en los modelos de producción deja patente el hecho de que cuanto más se apodera el comercio a gran escala de los circuitos alimentarios, transformándolos en circuitos mercantiles, tanto el acceso como la calidad de la alimentación se deterioran ( Friant, 2016).

De acuerdo a Zamora et al., (2015), Leff (2007), la cuestión ambiental más que una problemática ecológica, es una crisis del pensamiento y del entendimiento, de la ontología y de la epistemología con las que la civilización occidental ha comprendido el ser, a los entes y a las cosas; en esa línea, Power (2009), señala que probablemente nunca como en la actualidad la humanidad enfrenta el peligro de un deterioro tal en las condiciones ambientales que, de no reaccionar a tiempo, las futuras generaciones confrontaran criticas condiciones de supervivencia, coincide Valencia (2016) y señala que la gestión de la contaminación en los centros urbanos, especialmente la relacionada con aire, agua y suelo, impone enormes retos para la sociedad; el actual modelo de desarrollo económico, con patrones insostenibles de producción y consumo, ocasiona contaminación con altísimos costos para la sociedad, respecto a problemas de salud y deterioro de los recursos naturales renovables. Para De Burgos y Céspedes (2001), Ortega (2002), Correa (2004), Machin y Casas (2006), Ferán et al., (2016), el desafío medioambiental consiste en encontrar los niveles óptimos de contaminación, que surgen de comparar los beneficios que deriva la sociedad de las actividades que generan contaminación con los costos sociales que la contaminación genera. Al respecto Valencia (2016), determina que la ejecución de actividades económicas asociadas con la agricultura, la extracción de recursos pétreos, la disposición inadecuada de residuos sólidos orgánicos e inorgánicos y el cambio de uso del suelo para la construcción de viviendas son las causas principales que ocasionan riesgos e impactos ambientales a los elementos físicos, biológicos y socioculturales.

Un problema ambiental es la deforestación, cuyo problema no es la tala de por sí, sino el uso agrícola de las tierras que sigue a la deforestación, con la labranza mecánica, que trae como consecuencia una mineralización de la materia orgánica de los suelos, convirtiéndola en emisiones de dióxido de carbono. En el mismo sentido Ramírez (2016) considera que el no cambiar el uso actual del suelo, permite mantener los hábitats de muchas especies de animales y plantas, prevenir la erosión inducida por la deforestación y asegurar la recarga de los acuíferos. Con esto, la agricultura contribuye, en total, a casi $30 \%$ de las emisiones causantes del cambio climático (Correa, 2004), sobre el mismo tema De Lisio (2009), manifiesta que en general, de acuerdo a los datos de la Convención Marco de Naciones Unidas sobre Cambio Climático, la extracción de madera se convierte en Suramérica en una de las fuentes principales de los 1.700 millones de toneladas de $\mathrm{CO}_{2}$ que se descargan en la atmósfera terrestre, contribuyendo así al efecto invernadero, que en los últimos cien años confirman, que la temperatura media se ha incrementado de forma importante (Power, 2009).

La contaminación ambiental genera una deuda hacia la comunidad o región (Russi y Martinez, 2002), frente a los problemas ambientales ocasionados, la población intenta desarrollar una economía ecológica a partir de las limitaciones de la frontera agrícola (Pengue, 2010; Rodríguez, 2016); en este sentido, Pengue (2010), expresa que las externalidades, deberían ser consideradas para la compensación por la reducción de las áreas de producción agrícola como resultado de la intrusión salina, la degradación del suelo y el agotamiento de la disponibilidad a los recursos hídrico, entre otros. Así la protección ambiental es el resultado de la presión para internalizar los costos ambientales que se ponen de manifiesto con sus actividades productivas (Sinding, 2000).

Así también, los problemas ambientales ponen en riesgo la seguridad alimentaria (Stokstad, 2008). De acuerdo a Friedrich (2014), se considera que existe seguridad alimentaria cuando todas las personas tienen, en todo momento, acceso físico, social y económico a alimentos suficientes, inocuos y nutritivos que satisfacen sus necesidades energéticas diarias y preferencias alimentarias, para llevar una vida activa y sana. Por el contrario, un acceso nulo o incierto a los alimentos se ha definido como inseguridad alimentaria (Mundo et al., 2013). La opinión pública expresan que es inaceptable que en un mundo que produce 
suficiente alimento para todos, siga albergando a más de 850 millones de personas que pasan hambre, sin esperanza de cambiar la situación en toda su vida (Clementi y Vivero, 2006), al respecto Figueroa (2003), indica que la producción de alimentos ha crecido en muchos países; sin embargo, el número de personas hambrientas ha aumentado debido al rápido crecimiento de la población, la carencia de una distribución efectiva de alimentos, dada la vulnerabilidad de la cadena alimentaria a la contaminación ambiental. Friant (2016), indica que ante la severa crisis social y ambiental que el sistema alimentario enfrenta, los principios de comercio justo ayudan a imaginar una estructura económica alternativa que podría alimentar al mundo sin destruir la tierra. Por otra parte, Ezeta (2009), hace referencia a que en la India que es el tercer productor mundial de papa, la expansión del cultivo ocurrió significativamente en respuesta a decisiones políticas de sus gobiernos para aumentar la seguridad alimentaria, reducir la pobreza y mejorar la nutrición de sus habitantes, por lo que, la agricultura sería la mejor inversión para reducir pobreza (Ballarin, 2012).

En este contexto, para Ramírez (2016), el concepto de seguridad alimentaria debe diferenciarse del simple abastecimiento alimentario, pues éste sólo considera la disponibilidad de alimentos, independientemente de su procedencia, importaciones o producción, e implica riesgos graves y latentes, también es importante el acceso y el consumo de los mismos Council (2005); FAO (2008). De acuerdo a Friedrich (2014), en la actualidad, el mundo produce suficiente alimento para toda la población, se llega incluso, a desperdiciar, aproximadamente $30 \%$ de los alimentos producidos y se espera que la producción de alimentos se tenga que duplicar en los próximos 30 años. De acuerdo a (Clementi y Vivero, 2006), la lucha contra el hambre tiene un marcado carácter rural, lo que hace necesario programas que abarquen la producción, el acceso, la disponibilidad y la salubridad de los alimentos. Así, Carrion y Herrera (2012), expresan que la tendencia histórica de la agricultura en Ecuador ha sido la búsqueda de rentabilidad como fin último, lo que se opone a los objetivos nacionales de abastecimiento y suficiencia alimentaria. Según Friedrich (2014), en la última década se ha experimentado mayor variabilidad en la disponibilidad y acceso, por la falta física de alimentos, como por la volatilidad de precios debido a incidencias de sequías, inundaciones, plagas y enfermedades, como consecuencia de una mayor variabilidad climática, como efecto del cambio climático. Finalmente, Silva (2008), indica que la desigualdad de la Región de América Latina incluido Ecuador, la falta de oportunidades de empleo sigue siendo el principal problema que limita el acceso a los alimentos y otros bienes básicos de la población pobre.

En el cantón Guano de la provincia Chimborazo, Ecuador, la producción intensiva de monocultivos principalmente de papa han contribuido en gran medida al aparecimiento de varios problemas ambientales, que tienen relación con lo expuesto por De Lisio (2009), que indica agroecológicamente hablando, los sistemas intensivos de monocultivos niegan la posibilidad de alcanzar una agricultura adaptada a los ciclos biogeoquímicos terrestres, exigiendo por lo tanto, un alto subsidio humano, fertilizantes, herbicidas, fungicidas, etc., cuyos residuos, por lo general, se convierten en agentes de polución y contaminación. También Pengue (2010), indica que los sistemas de mono producción agrícola conllevan a una extracción selectiva de nutrientes del suelo, que lo agotan y fuerzan a una reposición vía fertilizantes minerales para recuperar la fertilidad actual, pero arrastran a crecientes niveles de contaminación y eutrofización. Así, las cuestiones ambientales como heladas, contaminación, sequía, erosión del suelo, entre otros, tienen influencia negativa en el cultivo de papa. En este contexto, la presente investigación tiene como objetivo realizar un estudio de las cuestiones ambientales que están afectando la producción de papa y su relación con la seguridad alimentaria.

\section{MATERIALES Y METODOS}

La presente investigación es de tipo no experimental, contempla un diseño transversal de recolección de información, se emplea un enfoque mixto de investigación a través de los métodos inductivo y deductivo para analizar la información recolectada, su alcance corresponde a una investigación de tipo descriptivo correlacional donde se explica la relación existente entre las cuestiones ambientales y la seguridad alimentaria a través del seguimiento del cultivo de papa. La población de estudio la componen los habitantes del sector rural del cantón Guano, considerando hombres y mujeres mayores de 12 años de edad. Para la selección de la muestra se aplicó el muestreo aleatorio simple. El tamaño de la muestra alcanzó a 380 individuos de una población de 35.093 habitantes del sector rural del cantón Guano, y se aplicó una encuesta empleando como instrumento un cuestionario que permitió recoger datos de las variables estudiadas. Trabajamos con un nivel de confianza del $95 \%$ y un nivel de significancia del $5 \%$. Para el contraste de la hipótesis de trabajo se aplicó una regresión lineal simple, que determina la correlación y determinación entre variables.

Las técnicas de investigación cualitativa empleadas fueron la observación de campo y la entrevista. En el primer caso se realizó un recorrido de las diferentes comunidades para constatar la presencia de cultivos de papa como reporta la teoría. En el caso de las entrevistas se optó por contactar a los líderes de las comunidades para llevar a cabo una entrevista no estructurada. El análisis de los datos de campo se realizó con el software estadístico SPSS. 


\section{RESULTADOS}

Dentro de la problemática ambiental de acuerdo a Fernández (2013), una de las mayores preocupaciones a nivel mundial es la influencia que ha tenido el calentamiento global y por tanto el cambio climático debido a la emisión de gases de efecto invernadero generados por un acelerado desarrollo económico asociado a un aumento de la población. Sobre este asunto ya se reportan impactos proyectados sobre los cultivos de seguridad alimentaria. Angulo (2012), expone que los impactos en los cultivos bajo el escenario expuesto para el 2030 donde la temperatura aumentaría hasta $1,3{ }^{\circ} \mathrm{C}$; y lluvias con variabilidad de más $15 \%$, indican que los rendimientos y el porcentaje de siembras perdidas no cambiarían, excepto donde ocurrirían disminuciones considerables de lluvias, los déficits de rendimientos variaran entre un 12 a $15 \%$, y el porcentaje de siembras perdidas de un 4 a $7 \%$ respecto a los valores actuales. De acuerdo a Noboa et al (2012), en el caso de la agricultura se evidencia impactos directos que inciden en el rendimiento de los cultivos y en los ciclos de crecimiento de las especies agrícolas, ocasionados principalmente por la variación de la temperatura y la presencia de algunas plagas e insectos que perjudican el normal desarrollo de los cultivos. Esto se relaciona con lo expuesto por Atlin (2017), que señala que el objetivo de los sistemas nacionales de semillas debería ser garantizar que la edad media de las variedades en los campos de los agricultores sea inferior a 10 años, tanto para asegurar que las ganancias genéticas se entregan constantemente a los agricultores y para mantenerse a la par con los efectos del cambio climático. Según Zamora et al., (2015), menciona que el crecimiento de la actividad humana está afectando la existencia de las comunidades bióticas al generar importantes pérdidas de especies y graves niveles de contaminación ambiental que se traducen en cambios drásticos en el funcionamiento y constitución de la biósfera.

Por otra parte, los Objetivos de Desarrollo Sostenible 2030 de las Naciones Unidas asignan una alta prioridad a la seguridad alimentaria para erradicar el hambre del mundo (Kline, 2017; Narayanan, 2015). La adaptación de los sistemas alimentarios, para disminuir el riesgo frecuente de las enfermedades y la salud es vital para garantizar la seguridad alimentaria (Cook y Frank, 2008). En este sentido, Friant (2016), presenta resultados de niveles de seguridad alimentaria mundial que indican que el $52,8 \%$ de los hogares están seguros, $23,3 \%$ con inseguridad leve, $14,3 \%$ con inseguridad moderada y $9,6 \%$ tienen una inseguridad alimentaria grave. En esa línea, Cafiero (2014), expresa la posibilidad de establecer un estándar mundial para medir la inseguridad alimentaria en los hogares y la población. A pesar del crecimiento poblacional, el incremento proyectado de la productividad agrícola para aumentar la producción de alimentos en un $70 \%$ para el 2.050, se proyectan mejoras en la seguridad alimentaria (Ghose 2014). El cambio climático tanto histórico como anticipado interactúa sustancialmente con los impulsores de las adquisiciones de tierras, ya que existe evidencia significativa de un efecto (negativo) de la pobreza asociado con la falta de acceso a los alimentos (Davis, 2015). Este planteamiento, permite de manera contextual introducirnos a los resultados obtenidos en la investigación.

\section{Descripción de la zona de estudio}

Según el censo poblacional del INEC (2010), la Agenda para la Transformación Productiva Territorial de la Provincia de Chimborazo (2011), y el Plan de Desarrollo y Ordenamiento Territorial del cantón Guano (2015), indican que este cantón tiene un área de $460,61 \mathrm{~km}^{2}$, se encuentra situado al norte de la provincia de Chimborazo, colindando al norte y oeste con la provincia de Tungurahua, al sur con el cantón Riobamba y al este con los cantones Pelileo y Penipe. El cantón Guano cuenta con 10 parroquias representando el $7,1 \%$ de la provincia de Chimborazo con una población de 42.900 habitantes, de los cuales el 18,2\% se encuentra en la zona urbana y el $81,8 \%$ en la zona rural, es decir 35.093 personas viven en el sector rural de este cantón. El 52,25\% de la población son mujeres mientras que 47,8 \% son hombres, el 53,3\% conforman la PEA, de esta población el $45,5 \%$ se dedica a la agricultura y ganadería mientras que el $15,7 \%$ de la población ocupada trabaja en industrias manufactureras. La pobreza en el cantón Guano es de 11.4 \% con relación a la provincia de Chimborazo. La escolaridad de la población en el cantón Guano es de 6,7 años para las mujeres y 7,7 años para los hombres.

El relieve del cantón es montañoso su rango de altitud va desde los 2.000 hasta los $6.310 \mathrm{msnm}$. Cuenta con $41,01 \%$ de superficie para la producción agrícola; el cultivo más representativo es la papa con un $28,31 \%$ de la superficie sembrada. La diversidad de pisos latitudinales genera una variedad de microclimas que permiten una diversidad de producción en esta región. El uso de tierras para el cantón Guano está distribuido en cultivos transitorios en un $58,1 \%$, pastos cultivados con $24,1 \%$, bosques $9,1 \%$, pastos naturales $4,4 \%$, cultivos permanentes $3,2 \%$, y un $1,1 \%$ de paramos. Por otro lado, la economía del cantón Guano es diferente en el sector urbano ya que es un importante centro artesanal de tejidos de lana, su especialidad es la elaboración de alfombras y artículos de cuero.

De acuerdo a la Memoria Técnica cantón Guano-SEMPLADES (2012), en el sector rural todas las parroquias se dedican fundamentalmente a la agricultura cuya problemática gira en torno a factores 
exógenos como el cambio climático afectado por la falta de lluvias, la merma en el caudal de los ríos, la tala indiscriminada de bosques, manejo inadecuado de desechos sólidos, monocultivo, exceso de pesticidas, falta de acceso a recursos financieros que respalden a los pequeños agricultores, problemas y cuidado del medio ambiente. En este cantón se ha evidenciado en los últimos años la disminución de la actividad agrícola y migración campo ciudad, esto se ve agravado en las dos terceras partes de la tierra de uso agrícola que sufren un proceso de deterioro que ha dado como consecuencia la baja productividad de varios cultivos, como es la papa. A nivel de la provincia se evidencia un deterioro superior del suelo en el cantón Guano, por su uso intensivo en cultivos transitorios, este mal uso se ve reflejado en escasos bosques primarios, vegetación natural, montes y paramos; y como consecuencia, el deterioro del medio ambiente.

\section{Análisis estadístico}

El análisis estadístico descriptivo refleja que la muestra está compuesta por $50,2 \%$ de hombres y $49,8 \%$ de mujeres; con un rango de edad de 14 a 59 años, con un promedio de edad de 26,62 años. Por otra parte, el $63 \%$ de la población se dedica al cultivo de papa, el 81,5\% de la población encuestada cultiva extensiones de tierra entre 1 a 5 Has y el 7,5\% tiene una extensión mayor a 10 Has de cultivo, lo que demuestra que existe un gran parcelamiento de la tierra. Según Ayaviri et al., (2016), en Ecuador la población tendrá mayores oportunidades a mejorar su calidad de vida cuando la seguridad alimentaria sea una política prioritaria para el Estado y sus instituciones donde aún no han incorporado algunos planteamientos realizados por organismos como la FAO sobre este tema.

El 94\% de la población encuestada expresa que los principales cambios ambientales percibidos en orden de mayor a menor importancia son la destrucción de páramos, el incremento de plagas y enfermedades en los cultivos, heladas frecuentes, contaminación por basura común, sequias prolongadas y la erosión del suelo. Las causas más importantes que han contribuido para que se produzcan estos cambios ambientales, son: el uso excesivo de pesticidas, falta de tratamiento de la basura doméstica, el monocultivo y la deforestación de acuerdo a la percepción del $72,6 \%$ de la población. Estos hallazgos coinciden con lo reportado por Monteros (2016), que indica que el principal problema en la producción de papa en Ecuador es la falta de agua, seguido por la mayor incidencia del tizón tardío phytophthora infestans, como la principal plaga que afecta de manera importante el rendimiento, en especial en la provincia de Chimborazo en la que se encuentra el cantón Guano, que tiene más del $90 \%$ de afectación en el cultivo de papa. Según Monteros (2016) la provincia de Chimborazo ocupa el tercer lugar de superficie sembrada de papa en el año 2014, con una participación del 17,55 \% a nivel nacional. A su vez, Salcedo y Lora (2002), menciona que los países andinos, entre ellos Ecuador, han sido objeto de diversos desastres naturales y climatológicos (sucesivas y prolongadas sequias, inundaciones, fuertes vientos, heladas, terremotos), que han puesto en jaque a la seguridad alimentaria de importantes grupos de la población.

El $61,4 \%$ de la población encuestada expresa que la producción de alimentos en el cantón Guano es muy buena, mientras que el $37,3 \%$ de la población expresa que la producción es mala. Por otro lado, el 66,7\% de población considera que existe suficiente producción de alimentos, mientras que el $33,3 \%$ de la población considera que la producción actual, no es suficiente para satisfacer las necesidades de los consumidores. En este contexto, para contrastar la hipótesis $(\mathrm{H} 1)$, del impacto negativo que producen los cambios ambientales sobre la producción de los cultivos de papa y la seguridad alimentaria de los habitantes del cantón Guano se emplea una regresión lineal simple para medir el grado de asociación de las variables de estudio. Para explicar la percepción que tienen las familias sobre el impacto de los cambios ambientales sobre el consumo de alimentos en el cantón Guano, se determinó que el $87,6 \%$ de la población considera que efectivamente los cambios ambientales producidos y sentidos en este cantón afectan la producción de papas.

Los resultados de la regresión lineal simple categórica determinaron que existe un bajo grado de relación entre los cambios ambientales percibidos y la producción de papa sobre la seguridad alimentaria de la población del cantón Guano, el coeficiente de correlación es muy bajo y no significativo $(R=0,07, p<$ 0,26 ). Estos datos se sustentan con los valores presentados en la (Tabla 1). Además se establece que existen otros factores exógenos que contribuyen con la seguridad alimentaria de esta localidad.

Tabla 1: Estadística de regresión

\begin{tabular}{|l|l|}
\hline Coeficiente de correlación múltiple & 0,073 \\
\hline Coeficiente de determinación $\mathrm{R}^{2}$ & 0,005 \\
\hline $\mathrm{R}^{2}$ ajustado & 0,001 \\
\hline Error Típico & 0,995 \\
\hline
\end{tabular}


Además indica que el efecto de regresión no es significativo para las variables referidas, dado que se obtuvo un $p<0,246$ (Tabla 2), el cual es mayor que el nivel crítico de comparación (alpha $\leq 0,05$ ), y por esta razón aceptamos la hipótesis nula que expresa que la baja producción de papa en el cantón Guano no pone en riesgo la seguridad alimentaria de sus habitantes. Esto se relaciona con lo que expresa Torres (2002), al referirse que la seguridad alimentaria como componente nacional se sustenta en que los desequilibrios en los espacios rurales han contribuido en la pobreza, y esto provoca tensiones de mayor explosividad al tratarse de una población más concentrada, que reclama mejores condiciones en su alimentación, y que la contaminación ambiental sea un componente a tener presente.

Tabla 2: Modelo de regresión

\begin{tabular}{|l|l|l|l|l|c|}
\hline & $\begin{array}{c}\text { Suma de } \\
\text { cuadrados }\end{array}$ & $\begin{array}{c}\text { Grados de } \\
\text { libertad }\end{array}$ & Media cuadrática & $F$ & Sig. \\
\hline Regresión & 1,260 & 1 & 1,260 & 1,256 & 0,264 \\
\hline Residual & 232,740 & 232 & 1,003 & & \\
\hline Total & 234,000 & 233 & & & \\
\hline
\end{tabular}

En relación a la producción de alimentos y el impacto de los cambios climáticos, el $11 \%$ de la población considera que existe un efecto negativo sobre el cultivo de papa. Las principales recomendaciones para reducir los impactos ambientales sentidos en el cantón Guano son: uso adecuado de los pesticidas con la tendencia a realizar un manejo de cultivos integrado y en lo posterior un manejo limpio, proporcionar incentivos y capacitación para cuidar el ambiente, recolección y clasificación de la basura doméstica, rotación de cultivos y conservación de bosques. En este contexto Sampaio (2009), indica que la capacidad de almacenamiento de agua del suelo y la capacidad de agua utilizable aumentó de forma considerable empleando el método de siembra directa en comparación con el tradicional laboreo del suelo; éste hecho representa un gran salto cualitativo en la capacidad de los suelos para resistir la desertización que es la base funcional de las cadenas alimenticias. Al relacionar los hallazgos, la población del cantón ha sentido los cambios ambientales a través de la baja producción de alimentos que se refleja en la volatilidad de los precios de los productos agrícolas en el mercado, en especial de la papa, lo que reduce el consumo de las familias más numerosas debido a que su capacidad de compra se ve afectada.

\section{DISCUSION}

Al comparar los periodos 1961 a 1963 con 2001 a 2003 la población mundial aumentó en un 98 \%, pero la producción de alimentos lo hizo en un $155 \%$ (Maletta, 2005). De acuerdo a Barrial y Barrial (2011), el crecimiento poblacional sumado al proceso de globalización que impone un nuevo modelo de alimentación, mediante las multinacionales que promocionan la comida rápida, el mal uso de los alimentos disponibles y la toma de decisiones incorrectas sobre el consumo de los mismos, estos cambios traen consigo hábitos alimentarios inadecuados y una serie de enfermedades, en este mismo sentido la pobreza es la causa fundamental de la inseguridad alimentaria ya que ella puede ser causa del desempleo 0 ingresos insuficientes que no permite adquirir los alimentos necesarios de forma suficiente (Figueroa, 2003). De acuerdo a Ballarin (2012), para el que hacer de la actividad rural, los procesos productivos deben ser fortalecidos y adaptarse a los cambios del contexto, incluyendo la tecnología, la integración de cadenas productivas y comercializadoras. El cultivo tradicional en la región andina del Ecuador, es la papa y uno de las más importantes en el mundo, este país es uno de los principales productores de Latinoamérica, ocupando el octavo lugar con 443 mil toneladas de producción por año FAO (2008). En la siguiente tabla se observa la producción de papa en la Provincia Chimborazo, de la que es parte el cantón Guano.

Como se aprecia en los datos de la Tabla 3, existe un descenso sostenido de la superficie sembrada a través de los años, variabilidad entre superficie sembrada y cosechada, y a su vez, un efecto directo sobre la productividad medida en $\mathrm{Tm} / \mathrm{Ha}$. Esta variación se relaciona directamente con factores ambientales y la presencia de plagas y enfermedades que reducen la productividad del cultivo de papa. Al comparar los resultados de la presente investigación, la población expresa que existe suficiente producción de alimentos en el cantón, en este sentido Ortega (2002), manifiesta que la producción, recolección, manipulación y distribución de alimentos hoy en día en Ecuador, viene determinada por una dinámica economicista donde importa poco la calidad de lo que comemos, ni el contexto en el que son producidos los alimentos. Hoy en día muchas empresas del sector agrícola no consideran la contaminación que puede generar en la alimentación, y de acuerdo a Russi (2002), si pueden ser considerados como costos al conjunto de daños que transfieren a la colectividad, probablemente los daños ambientales producidos se reducirían, y la calidad de los alimentos mejoraría. 
Tabla 3: Producción de papa en la provincia de Chimborazo (Datos tomados de ESPAC, 2011-2015)

\begin{tabular}{|c|c|c|c|c|}
\hline Año & $\begin{array}{c}\text { Superficie } \\
\text { Sembrada }(\mathrm{Ha})\end{array}$ & $\begin{array}{c}\text { Superficie } \\
\text { Cosechada }(\mathrm{Ha})\end{array}$ & $\begin{array}{c}\text { Producción Total } \\
\text { Tm }\end{array}$ & $\begin{array}{c}\text { Rendimiento } \\
\text { Tm/Ha }\end{array}$ \\
\hline 2011 & 11378 & 11090 & 47882 & 4,31 \\
\hline 2012 & 10232 & 10015 & 50657 & 5,05 \\
\hline 2013 & 15271 & 14848 & 80766 & 5,43 \\
\hline 2014 & 6279 & 6184 & 85053 & 13,75 \\
\hline 2015 & 4386 & 3867 & 43131 & 11,15 \\
\hline
\end{tabular}

Al analizar la tabla número 3 se explica que existe una reducción del $58,88 \%$ de la superficie sembrada de papa del año 2013 al 2014 que equivale a $8992 \mathrm{Ha}$ menos de siembra, para el año 2015 tenemos una disminución del 30,15\%, que equivale a 1893 Has menos de cultivo. Por otro lado el rendimiento se incrementó en 8,32 Tm/Ha del 2013 al 2014, mientras que el 2015 hubo una reducción del rendimiento por Ha de 2,6 Tm. De acuerdo a Monteros (2016), esta mayor productividad se debe a la utilización de semilla superchola, mayor densidad de siembra, una fertilización compuesta, mecanización para la preparación del suelo y alguna infraestructura de riego.

Con relación a los cambios ambientales percibidos en el cantón Guano, los encuestados consideran como los más importantes a las sequias, heladas, incremento de la presencia de plagas y enfermedades en los cultivos, destrucción de paramos y contaminación del ambiente por basura común, este tipo de contaminación según Leff (2007), son las más comunes en cultivos locales, han destruido ciertos cultivos y es necesario buscar alternativas de solución porque pueden ser perjudiciales para la salud (Valencia, 2016). Respecto a las causas que producen cambios ambientales en el cantón Guano, los resultados indican como los más importantes al monocultivo, uso excesivo de pesticidas, falta de tratamiento de la basura común y la deforestación; sin duda, el uso excesivo de pesticidas pueden modificar los sistemas de cultivo (Ortega, 2002) y modifican hábitos de consumo de alimentos Franceschi (2014), junto a la falta de conciencia en la buena administración de los residuos orgánicos e inorgánicos (Frontons, 2006).

Los problemas ambientales como consecuencia de la contaminación provocada abordan efectos negativos en la población provocando deterioro en los suelos, deficiencias en el acceso a los alimentos y problemas de salud (Ferán, 2016), aunque no todos los efectos del cambio climático pueden resultar negativos, la mayoría de los sectores necesitarán encontrar maneras de lidiar con los efectos. Las áreas de alto riesgo son la agricultura y la seguridad alimentaria. En base a los resultados obtenidos se desprende nuevos cuestionamientos e hipótesis a resolver como la diferencia en la seguridad alimentaria entre los habitantes del sector urbano y el sector rural, entre poblaciones de la costa, sierra y la amazonia entre otros.

\section{CONCLUSIONES}

Los cambios ambientales más importantes sentidos en el cantón Guano en los últimos años son la destrucción de páramos, incremento de plagas y enfermedades en los cultivos que inciden sobre la producción de papa, pero no sobre la seguridad alimentaria.

Las causas más importantes de los cambios ambientales son el uso excesivo de pesticidas, falta de tratamiento y clasificación de la basura, monocultivo y deforestación. Sobre estos factores se deberían enfocar las prácticas de remediación ambiental para producir alimentos de una manera sustentable que permita garantizar el abastecimiento de alimentos para las siguientes generaciones y así asegurar una producción local y el acceso a los alimentos de forma permanente.

Con las relaciones establecidas entre las variables de estudio, se determina que la seguridad alimentaria no se ve comprometida por la baja producción de papa, ya que existen otros mercados locales en los cuales los habitantes del cantón tienen acceso a los alimentos en todos los periodos y estaciones del año para satisfacer las necesidades alimentarias.

\section{REFERENCIAS}

Agenda para la Transformación Productiva Territorial Provincia de Chimborazo, Ministerio de Coordinación de la Producción Empleo y Competitividad, 1 (1), 1-83 (2011) 
Angulo, L., Programa de Adaptación al Cambio Climático PACC- Perú, Servicio Nacional de Meteorología e Hidrología, (12), 1-26 (2012)

Atlin, G.; Cairns, J.; Das, B., Rapid Breeding and Varietal Replacement are Critical to Adaptation of Cropping Systems in the Developing World to Climate Change, http://dx.doi.org/10.1016/j.gfs.2017.01.008, Global Food Security, 12(1), 31-37 (2017)

Ayaviri, D., Avances y Progresos de las Políticas y Estrategias de la Seguridad Alimentaria Ecuador, https://goo.gl/kZGspG, ISSN: 2306-8582, Revista Investigación Altoandina, 18(2), 213-222 (2016)

An Introduction to the Basic Concepts of Food Security, https://goo.gl/dSdTHn, Food Security Programme, FAO (2008)

Ballarin, A., La Crisis Alimentaria Mundial, Retos y Soluciones, icam-es@arrakis.es, Instituto de Cuestiones Agrarias y Medioambientales, 1-5 (2012)

Barrial, A. y Barrial, A., La Educación Alimentaria y Nutricional desde una Dimensión Sociocultural como Contribución a la Seguridad Alimentaria y Nutricional, angelicam@fcsh.upr.edu.cu, Contribuciones a las Ciencias Sociales, 1-23 (2011)

Cafiero, C.; Quiñonez, H.; Ballard, T.; Kepple, A., Validity and Reliability of Food Security Measures, doi: 10.1111 /nyas.12594, Annals of the Ney York Academy of Sciences, 1331, 230-248 (2014)

Cook, J. y Frank, D., Food Security, Poverty, and Human Development in the United States, doi: 10.1196/annals.1425.001, Publimed, 1136, 193-209 (2008)

Correa, F., Crecimiento Económico y Medio Ambiente: Una Revisión Analítica de la Hipótesis de la Curva Ambiental de Kuznets, https://goo.gl/Gj5JTp, ISSN 1692-3324, Semestre Económico, 6(10), 11-30 (2007)

Council, N. R., Measuring Food Insecurity and Hunger: Phase1 Report. Panel to review U.S. Department of Agricultures Measurement of Food Insecurity and Hunger, https://goo.gl/hzoy4L, National Academies of Sciences Engineering Medicine (2005)

Clementi, L. y Vivero, J., La Nueva Seguridad Alimentaria en América Latina y la Lucha Contra el Hambre en Guatemala, FAO, (8), 249-272 (2006)

Davis, K.; Rulli, M.; D'Odorico, P., The Global Land Rush and Climate Change, doi: 10.1002/2014EF000281, Agu-publications, 3, 298-311 (2015)

De Burgos, J. y Céspedes, L., La Protección Ambiental y el Resultado, Un Análisis Crítico de su Relación, Investigaciones Europeas de Dirección y Economía de la Empresa, 7 (2), 93-108 (2001)

De Lisio, A., La Cuestión Ambiental como Dilema de Desarrollo en el Mercosur, https://goo.gl/rqceFx, ISSN: 1012-1617, Revista Geográfica Venezolana, (1), 11-33 (2009)

Ezeta, F., Una Visión de la Expansión de la Papa en el Asia, F.EZETA@cgiar.org, Revista Latinoamericana de la Papa, 15(1), 1-11 (2006)

Encuesta de Superficie y Producción Agrícola Continua. ESPAC, (2011 a 2015)

Ferán, A. y otros tres autores., La Contaminación Ambiental desde el Punto de Vista de la Economía, https://goo.gl/w85gbT, ISSN: 2254-7630, Iconos Revista Caribeña de Ciencias Sociales, (1), 1-18(2016)

Fernández, M., Efectos del Cambio Climático en la Producción y Rendimiento de Cultivos por Sectores, Fondo Financiero de Proyectos de Desarrollo, (1), 1-50 (2013)

Figueroa, D., Seguridad Alimentaria Familiar, dixisnut@plopmail.com, Revista Salud Pública y Nutrición, (2), 1-9 (2003) 
Franceschi, F., Ambiente: Nueva Cuestión Social para el Trabajo Social, https://goo.gl/BPHzvs, ISSN: 04825276, Revista de Ciencias Sociales, (143), 1-11(2014)

Friant, M., Comercio Justo, Seguridad Alimentaria y Globalización: Construyendo Sistemas Alimentarios Alternativos, doi: http://dx.doi.org/10.17141/iconos.55.2016.1959, Iconos Revista de Ciencias Sociales, (55), 215-240 (2016)

Friedrich, T., La Seguridad Alimentaria: Retos Actuales, https://goo.gl/JXtdt3, Revista Cubana de Ciencia Agrícola, 48(4), 319-322 (2014)

Frontons, G., La Cuestión Agrícola, https://goo.gl/U2QecE, ISSN: 0329-3475, Universidad del Centro Educativo Latinoamérica, (2), 2-13 (2006)

Ghose, B., Food Security and Food Self- Sufficiency in China: From past to 2050, doi: 10.1002/fes3.48, Food and Energy Security, 3(2), 86-95 (2014)

Instituto Nacional de Estadísticas y Censos (INEC), (2010)

Kline, K. y otros diez autores. Reconciling Food Security and Bioenergy: Priorities for Action, doi: 10.1111/gcbb.12366, Global Change Biology Bioenergy, 9, 557-576 (2017)

Leff, E., La Complejidad Ambiental, https://goo.gl/HKgK1h, ISSN: 0717-6554, Revista de la Universidad Bolivariana, (16), 1-9 (2007)

Machin, M. y Casas, M., Valoración Económica de los Recursos Naturales: Perspectiva a través de los Diferentes Enfoques de Mercado, Revista Futuros, (13), 1-9 (2006)

Maletta, H., Tendencias Mundiales en la Seguridad Alimentaria, https://goo.gl/35U56w, Instituto de Investigación en Ciencias Sociales, (13) 1-69 (2005).

Monteros, A., Rendimientos de Papa en el Ecuador Segundo Ciclo 2015, amonteros@magap.gob.ec, Dirección de Análisis y Procesamiento de la Información, 1-9 (2016)

Mundo, V. y otros tres autores, Epidemiologia de la Inseguridad Alimentaria en México, https://goo.gl/BAs2tN, 2:S206-S213, Instituto Nacional de Salud Pública de México, (2) ,06-213 (2013)

Narayanan, S., Food Security in India: The Imperative and Its Challenges, doi: 10.1002/app5.62, Asia \& the Pacific Policy Studies, 2(1), 197-209 (2015)

Noboa, S. y otros tres autores, Impacto del Cambio Climático en la Agricultura de Subsistencia en el Ecuador, https://goo.gl/5FUUmN, ISSN: 1885-9135, Serie Avances de Investigación, (66), 2-95 (2012)

Ortega, J. y otros cuatro autores, Contaminantes Medio Ambientales en la Alimentación, Grupo de Trabajo de Salud Medio Ambiental de la Sociedad Valenciana de Pediatría, (1), 1-8 (2002)

Plan de Desarrollo y Ordenamiento Territorial cantón Guano, https://goo.gl/VB214o, (2015)

Pengue, W., Agua Virtual, Agronegocio Sojero y Cuestiones Económico Ambientales Futuras, wapengue@sinectis.com.ar, Grupo de Ecología del Paisaje y Medio Ambiente, 1-16 (2010)

Power, P., El Calentamiento Global y las Emisiones de Carbono, https://goo.gl/onKuY6, ISSN 1025-9929, Red de Revistas Científicas de América Latina, el Caribe, España y Portugal, (27), 101-122 (2009)

Ramírez, M., Lineamientos para Seguridad Alimentaria: Retos y Perspectivas, Fundación Universidad Autónoma de Colombia, (1), 2-14 (2016)

Russi, D. y Martinez, J., Los Pasivos Ambientales, https://goo.gl/t8eb1N, ISSN: 1390-1249, Iconos Revista de Ciencias Sociales, (15), 123-131 (2002) 
Salcedo, S. y Lora, A., Los Problemas de la Seguridad Alimentaria desde la Perspectiva Regional Andina, https://goo.gl/Z6dncz, Políticas de Seguridad Alimentaria en los Países de la Comunidad Andina, (11), 116 (2002)

Sampaio, E., Estudio de las Prácticas Culturales en sus Relaciones con Agricultura, Suelo Y Ambiente, doi: 10.1612/inf.tecnol.4003it.08, Información Tecnológica, 20(3) ,113-123 (2009)

Secretaria Nacional de Planificación y Desarrollo SEMPLADES, Generación de Geo información para la Gestión del Territorio a Nivel Nacional Escala 1:25.000. Memoria Técnica Canton Guano (2012)

Silva, J. y otros tres autores, Estrategias de Desarrollo, Política Pública y Seguridad Alimentaria en América Latina y el Caribe, https://goo.gl/2hfhZd, Centro Latinoamericano para el Desarrollo Rural, (18), 1-43 (2008)

Stokstad, E., Duelling Visions for a Hungry World, doi: 10.1126/science.319.5869.1474, Science, 319, 14741476 (2008)

Valencia, E. y otros tres autores, Recuperación Ambiental y Bienestar Social en México: El Caso de Calamaya Estado de México, https://goo.gl/shTxSF, Revista DELOS, 25, 1-21(2016)

Zamora, M., Cambio Global: El Antropoceno, https://goo.gl/w4FctL, ISSN 1405-0269, Ciencia Ergo Sum, 23, 67-75 (2015) 